\title{
Development of a Video Analysis Software for Biomechanics Education
}

Dr. Hirohito Kobayashi, University of Wisconsin, Platteville

University of Wisconsin-Madison Ph.D. University of Wisconsin-Madison, M.S. Waseda University, Tokyo, JAPAN, B.S. 


\section{Work-In-Progress: Development of a Video Analysis Software for Biomechanics Education}

\section{Introduction}

In the undergraduate senior-level biomechanics course, students should learn both 1) tissue mechanics through stress-strain analysis that requires a tissue mechanical testing or a simulation based on Finite Element Analysis (FEA) and 2) biomechanics of body motion that requires the motion tracking system. However, setting up these two types of experiments can lead to a significant financial investment in the laboratory. Worst of all, it is often very challenging to synchronize data collected from different types of experiments. Without the properly synchronized data collected from tissue mechanical test and body motion analysis, the students will have difficulty understanding the causality between the biomechanics of body motion and tissue mechanics A successful example of the implementing FEA simulation in tissue biomechanics lecture ${ }^{1}$ or the introduction of a digital image motion analysis in the lecture to cover biomechanics of body motion ${ }^{2}$ were reported in the past. However, the author is not aware of any study reporting the efficacy of the teaching both biomechanics of body motion and tissue mechanics in one biomechanics course based on the concept of a student-centered hands-on laboratory by using a video image analysis software.

To resolve the challenge confronting the biomechanics course, Virtual Mechanics Laboratory (VML) software ${ }^{3,4}$, a digital video image analysis software, was improved with the addition of the Data Matching module for the application of biomechanics education. VML allows users to analyze digital video images captured from a moving body and/or deforming tissues, with a digital image tracking algorithm named "Digital Image Correlation" (DIC) ${ }^{5,6}$. Originally, VML was created as a supplemental virtual "hands-on" experimental tool for Dynamics and Mechanics of Material courses, yet it was missing the data matching feature that was essential for biomechanics laboratory that bridges tissue mechanics and biomechanics of body motion. Hence the Data Matching Module was added to VML for the students to understand the causality between tissue mechanics and biomechanics of body motion through the matched data.

The improved VML will be tested in the brand-new Biomechanics course to be offered at the University of Wisconsin-Platteville in Fall 2020. This Biomechanics course covers 1) Statics and Dynamics applied to biomechanics, 2) Application of mechanics of material in tissue mechanics, 3) Introduction to digital image motion tracking and VML. This paper presents the concept of a Biomechanics course developed around VML that aims to achieve hands-on virtual laboratory experience.

\section{Video analysis steps in Virtual Mechanics Laboratory (Fig.1)}

The video analysis with VML consists of the following steps.

1) Video crop with Video Edit module: In this module, users can crop the captured video to a proper length before the analysis conducted in the following modules. When a video image of a moving object is recorded by a digital camera in the field, it is notably difficult to record only the key motion part. As a result, the captured video image often includes segments of the non-essential motion that should be removed in the Edit module.

2) Motion analysis with Dynamics module: User can conduct object tracking with the DIC algorithm. Multiple objects can be selected with simple mouse-click actions. The measured kinematic information can be exported as EXCEL® format data Finally, students will be 
tasked to evaluate the unknown information (Acceleration, initial velocity, etc.) by applying the formula to the exported data.

3) Deformation analysis with Mechanics of Material module: User can select a Region of Interest (ROI) in the video image (digital camera and/or ultrasound videos) captured from deforming tissues for evaluating the tissue displacements and deformations. The evaluated data can be exported for further analysis. Students will be tasked to evaluate the unknown information such as the stress-strain relation by applying the formula to the processed data.

4) Data Matching module: User can match (synchronize) data collected from different testing devices with Cross-Correlation technique ${ }^{7}$. For example, the tissue strain information deduced from the Mechanics of Material module may be synchronized with stress information measured and evaluated from a mechanical testing machine in this module.

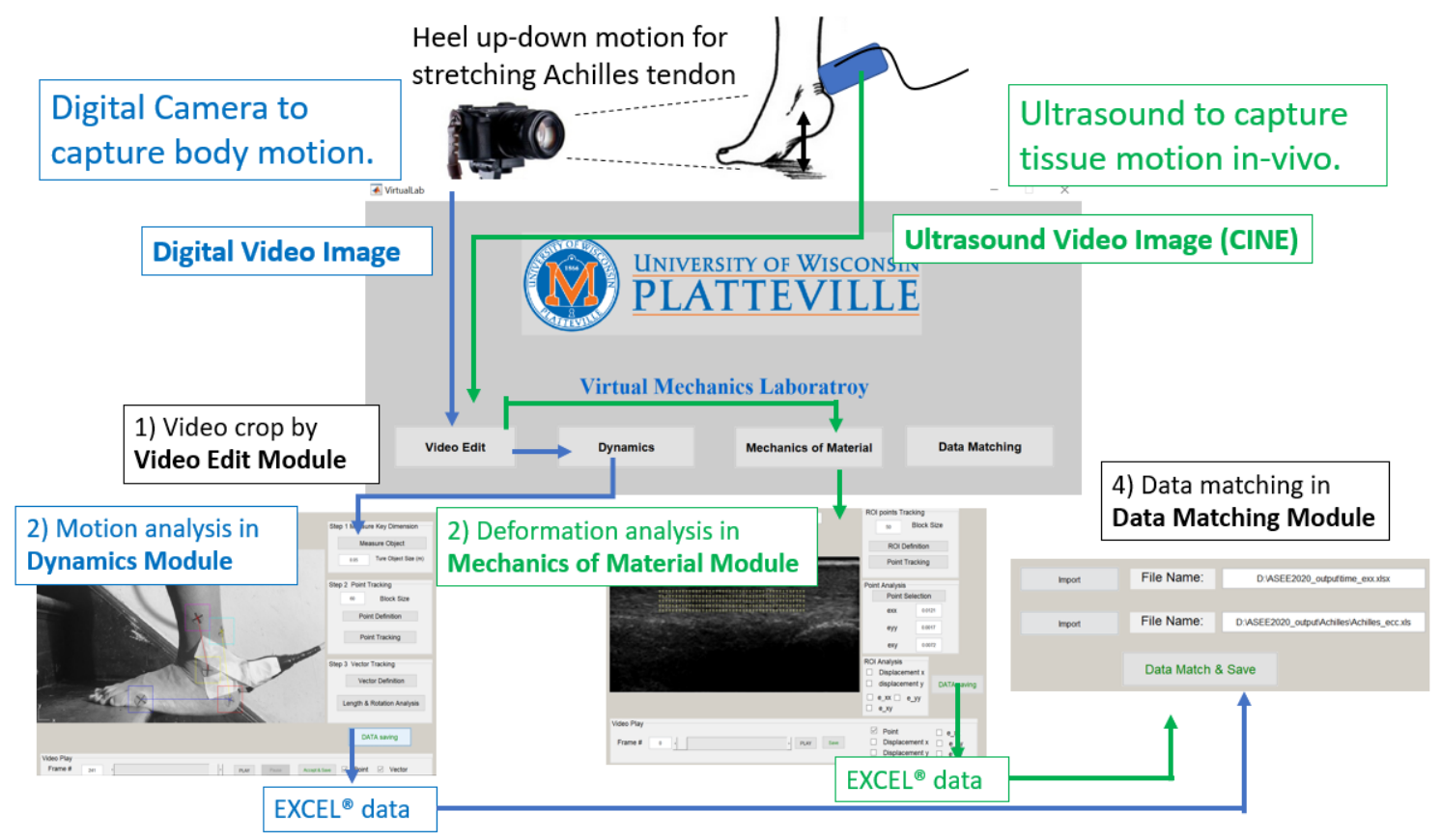

Fig. 1. Video analysis steps in Virtual Mechanics Laboratory

\section{Biomechanics projects with Virtual Mechanics Laboratory}

The following projects may be used for the laboratories in our Biomechanics course. However, students are allowed to pick any topics by submitting a simple proposal with the justification.

1) Sports Biomechanics (Fig.2): In this project, students will be tasked to capture and analyze the video images of body motions in any sports that involve impacts such as ball-kicking motion by a soccer player. The impact information (Coefficient of Restitution, etc.) shall be evaluated by using the kinematic information deduced from the Dynamics module.

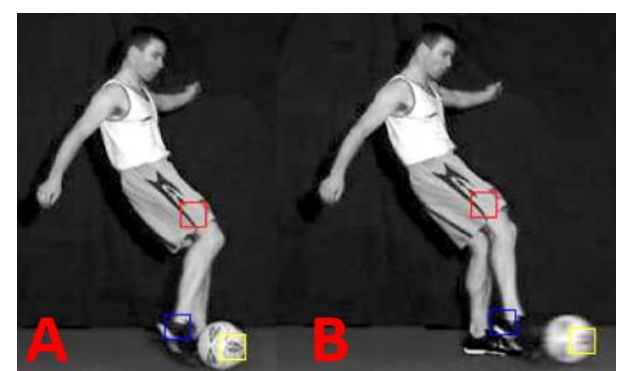

Fig. 2. Tracked video frames of the ball kick The foot (BLUE), ball(YELLOW), and the logo(RED) on pants are tracked. The pre- and post-ball-kick positions are shown in Fig.2A and 2B respectably.

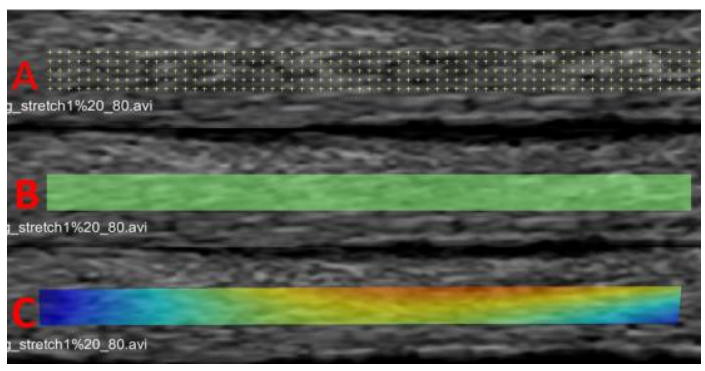

Fig. 3. ROI and strain map of a porcine tendon under stretch A: Tracked points in a rectangular-shaped ROI

B: $0 \%$ strain (GREEN) measured at rest (frame \#1)

$\mathrm{C}$ : Non-uniform strain distribution measured at $1 \%$ max strain 
2) Mechanics of a biological tissue in-vitro (Fig.3): In this project, students will be tasked to capture ultrasound images of a porcine digital flexor tendon stretched by a mechanical tester. The stress-strain relation will be evaluated by matching the strain deduced from Mechanics of Material module and the load recorded by the tester in the Data Matching module.

3) Mechanics of a biological tissue in-vivo (Fig.1,4,5): This project aims to evaluate the in-vivo stress-strain relation of the Achilles tendon during the heel-up-down motion. First, students are tasked to capture the video image of heel-up-down motion and the ultrasound video image of a gradually stretching Achilles tendon with a digital camera and ultrasound system respectively. The in-vivo stress will be evaluated with the following information.

- Tension force $T$ applied Achilles calculated from the Free-Body-Diagram of a foot (Fig.4A). All angles are measured through foot motion tracking (Fig.4B).

- The deformation-dependent cross-section area will be evaluated by using the initial crosssectional area measured by ultrasound and the assumption of tissue incompressibility. Average tissue strain will be measured through ROI analysis conducted in the Mechanics of Material module (Fig.5). Finally, in-vivo stress and strain will be matched in the Data Matching module for evaluation of stress-strain relation.

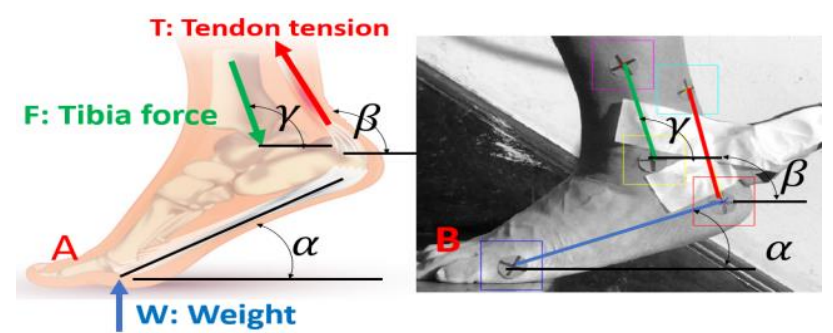

Fig. 4. Foot Free-Body-Diagram (FBD) and the tracked foot A: FBD of a foot: Tibia force $F$ and the tendon tension $T$ will be evaluated by the equations of equilibrium.

B: Tracked foot: The angles $(\alpha, \beta, \gamma)$ for solving equations of equilibrium will be measured by motion tracking.

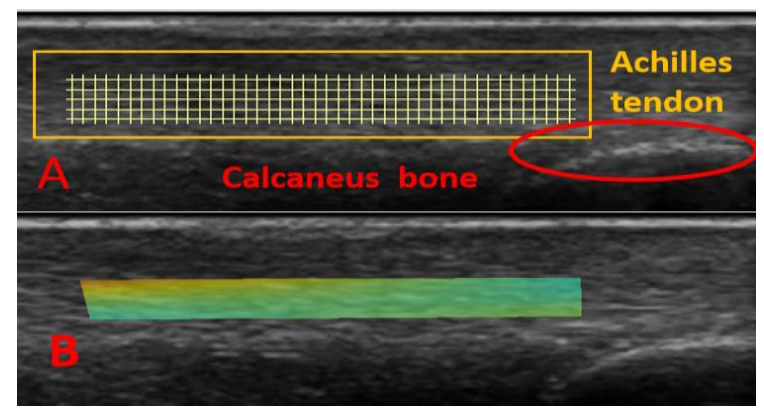

Fig. 5. ROI and strain map of a stretched Achilles tendon A: Tracked points in the rectangular-shaped ROI B: Strain color map measured at a max stretch

\section{Assessing the efficacy of the biomechanics laboratories with VML}

Student achievement will be assessed with the quiz, homework, project report, and final exam. Since this is a brand-new course, it is not possible to statistically compare the test scores/grades of this course as the experimental group and the course without VML as the control group to verify the efficacy of the biomechanics laboratories with VML. Hence, the following two tools will be implemented as alternative assessment tools.

Quiz: Conceptual quizzes will be issued before and after each laboratory and compared.

Student survey: Feedback from students will be collected by the end-of-the-semester survey prepared specifically for this course.

\section{References}

1) https://peer.asee.org/integrating-finite-element-analysis-into-an-undergraduate-biomechanics-course

2) https://peer.asee.org/new-course-development-biomechanics-and-biomaterials-for-mechanical-engineering-students

3) https://peer.asee.org/development-of-motion-analysis-software-for-dynamics-education

4) https://www.asee.org/public/conferences/64/papers/15984/view

5) T. C. Chu, W. F. Ranson, M. A. Sutton, W. H. Peters, "Applications of digital-image-correlation techniques to experimental mechanics," Exp. Mech., vol. 25, pp.232-244, 1985

6) H. A. Bruck, S. R. McNeill, M. A. Sutton, W. H. Peters III, "Digital image correlation using Newton-Raphson method of partial differential correction," Exp. Mech., vol.29, pp.261-267, 1989

7) Buck, John R., Michael M. Daniel, and Andrew C. Singer, Computer Explorations in Signals and Systems Using MATLAB ${ }^{\circledR}$. 2nd Edition. Upper Saddle River, NJ: Prentice Hall, 2002. 